\title{
Absence of superconducting dome at the charge-density-wave quantum phase transition in $2 \mathrm{H}-\mathrm{NbSe}_{2}$
}

\author{
Owen Moulding, ${ }^{1}$ Israel Osmond, ${ }^{1}$ Felix Flicker, ${ }^{2,3,4}$ Takaki Muramatsu, ${ }^{1}$ and Sven Friedemann $\odot^{1, *}$ \\ ${ }^{1}$ HH Wills Laboratory, Tyndall Avenue, University of Bristol, Bristol, BS8 1TL, United Kingdom \\ ${ }^{2}$ Rudolf Peierls Centre for Theoretical Physics, University of Oxford, Department of Physics, Clarendon Laboratory, \\ Parks Road, Oxford, OX1 3PU, United Kingdom \\ ${ }^{3}$ School of Physics and Astronomy, Cardiff University, Cardiff CF24 3AA, United Kingdom \\ ${ }^{4}$ School of Mathematics, University of Bristol, Bristol BS8 1TW, United Kingdom
}

(Received 15 June 2020; revised 11 November 2020; accepted 25 November 2020; published 18 December 2020)

\begin{abstract}
Superconductivity is often found in a dome around quantum critical points, i.e., second-order quantum phase transitions. Here, we show that an enhancement of superconductivity is avoided at the critical pressure of the charge-density-wave (CDW) state in $2 \mathrm{H}-\mathrm{NbSe}_{2}$. We present comprehensive high-pressure Hall effect and magnetic susceptibility measurements of the CDW and superconducting state in $2 \mathrm{H}-\mathrm{NbSe}_{2}$. Initially, the second-order CDW transition is suppressed smoothly but it drops to zero abruptly at $P_{\mathrm{CDW}}=4.4 \mathrm{GPa}$ thus indicating a change to first order, while the superconducting transition temperature $T_{\mathrm{c}}$ rises continuously up to $P_{\mathrm{CDW}}$ but is constant above. The putative first-order nature of the $\mathrm{CDW}$ transition is suggested as the cause for the absence of a superconducting dome at $P_{\mathrm{CDW}}$. Indeed, we show that the suppression of the superconducting state at low pressures is due to the loss of density of states inside the CDW phase, while the initial suppression of the CDW state is accounted for by the stiffening of the underlying bare phonon mode.
\end{abstract}

DOI: 10.1103/PhysRevResearch.2.043392

\section{INTRODUCTION}

The interplay of competing orders is of fundamental and practical interest [1-4]. Controlled switching between phases promises new applications in data storage and sensing [1]. On a fundamental level, understanding the interplay between ground states provides important insight into the mechanism underlying each ground state and can reveal new phenomena at the border of ordered phases [5-7]. For instance, a large body of work focuses on the interplay of superconductivity and charge order in cuprate high-temperature superconductors [8].

With both superconductivity and charge-density-wave (CDW) order stabilised by the opening of a gap on (parts of) the Fermi surface, a mutual competition between the two states has been anticipated since early studies [9]. As an alternative, superconductivity in a dome around quantum critical points was suggested to be promoted by quantum fluctuations of the ordered state with prominent examples in heavy-fermion antiferromagnets [5], CDW systems [10], and the CDW and pseudogap order in cuprate superconductors [11]. In addition to competition and promotion, superconductivity and charge order can coexist for instance by opening a

\footnotetext{
*Sven.Friedemann@ bristol.ac.uk

Published by the American Physical Society under the terms of the Creative Commons Attribution 4.0 International license. Further distribution of this work must maintain attribution to the author(s) and the published article's title, journal citation, and DOI.
}

gap on different parts of the Fermi surface; $2 \mathrm{H}-\mathrm{NbSe}_{2}$ is a prototypical material hosting both CDW order and superconductivity. However, fundamental questions about the interplay of the CDW and superconductivity remain open.

The interplay of CDW order and superconductivity in $2 \mathrm{H}-\mathrm{NbSe}_{2}$ remains disputed $[12,13]$. CDW order sets in at $T_{\mathrm{CDW}} \sim 33 \mathrm{~K}$, while superconductivity is present below $T_{\mathrm{c}}=7.1 \mathrm{~K}$ at ambient pressure [14-17]. Superconductivity opens gaps of different sizes on most of the Fermi surface, while the CDW opens a gap on small parts of the zone-corner niobium-derived Fermi surface sheets only $[12,13,15,18,18$ 21]. The separation of the CDW and superconducting gaps in $k$ space was interpreted as a hallmark for coexistence of the two ordered states. In addition, some studies suggested that superconductivity is boosted by the static CDW order [12], while further studies suggested a promotion of superconductivity by the soft modes present at the quantum critical point of the CDW order [22,23]. Finally, some studies suggested a bidirectional competition for density of states between the CDW and superconductivity [13,23-25]. Here, we use comprehensive high-pressure tuning of the CDW and superconducting states to reveal the absence of a superconducting dome thus ruling out a promotion of superconductivity by soft modes of the CDW. Rather, we show very clearly that superconductivity is reduced inside the CDW phase because of the loss of density of states. At the same time, we find indications of a first-order CDW transition which is likely to be the reason for the absence of a domeshaped superconducting phase at the critical pressure of the CDW. 


\section{EXPERIMENTAL METHODS}

\section{A. Samples}

$2 \mathrm{H}-\mathrm{NbSe}_{2}$ samples were grown by J. A. Wilson [16] using the vapour transport method and have a high residual resistivity ratio, $\rho(T=300 \mathrm{~K}) / \rho(T=9 \mathrm{~K})>60$, confirming the good crystal quality. Samples were cut with a scalpel. Lateral sample dimensions have been measured with an optical microscope at ambient pressure [see inset in Fig. 3(a)], the sample thickness, $t$, was estimated from the sample mass and the lateral dimensions using the known density of $2 \mathrm{H}-\mathrm{NbSe}_{2}$. The uncertainty of $10 \%$ for $t$ results in a systematic relative uncertainty of the Hall coefficient of the same amount. The magnetic field was applied along the crystallographic $c$ direction.

\section{B. High-pressure measurements}

High-pressure measurements used moissanite anvils cells with a culet size of $800 \mu \mathrm{m}$ for both the electrical and magnetic measurements. Both types of measurement used metallic gaskets which were prepared by indenting $450-\mu$ m-thick $\mathrm{BeCu}$ to approximately $60 \mu \mathrm{m}$ followed by drilling a 450- $\mu \mathrm{m}$ hole.

Pressure was determined at room temperature by ruby fluorescence, with multiple ruby flakes placed within the sample chamber as a manometer. The uncertainty of the pressure is taken as the standard deviation between pressure estimates from rubies across the sample chamber, both before and after a measurement. A comparison with the pressure obtained from ruby at room temperature and the superconducting transition of a piece of lead revealed good agreement to within $0.2 \mathrm{GPa}$ for the pressure cells used for magnetization measurements.

The effect of different pressure media is discussed in S II of the Supplemental Material Ref. [26].

\section{Electrical transport measurements}

For the electrical measurements, six bilayer electrodes were deposited on one anvil in a three-step process without breaking vacuum. Firstly, the anvil was cleaned using an RF argon plasma etch, followed by sputtering $20 \mathrm{~nm}$ of nichrome, and finally evaporation of $150 \mathrm{~nm}$ gold. To remove potential electrical shorts between electrodes, any nichrome overspray was removed using TFN etchant.

Gold contacts were evaporated on top of the sample. EpoTek H20E silver paint was used to connect the samples to the electrodes on the anvil. A four-probe AC method was used to measure the resistance with a current $I=1 \mathrm{~mA}$. The six electrodes were used to measure $V_{1}$, the longitudinal and $V_{\mathrm{t}}$, the transverse voltages, respectively. The Hall coefficient was calculated from the antisymmetric part of $V_{\mathrm{t}}(H)$ under reversal of the magnetic field $H$ as

$$
R_{\mathrm{H}}=\frac{V_{\mathrm{t}}(H)-V_{\mathrm{t}}(-H)}{2 \mu_{0} H} \frac{t}{I} .
$$

For the electrical measurements, the gaskets were insulated using a mixture of Stycast epoxy 2850FT and BN powder; the mixture was pressed between the anvils to above the maximum pressure required for the experiments and then cured, while pressurised. A $400-\mu \mathrm{m}$ hole through the insulation was drilled for the sample space.

\section{Magnetic measurements}

A Quantum Design Magnetic Property Measurement System (MPMS) was used to measure the DC magnetic moment of the sample inside the pressure cell as detailed in Sec. IV of the Supplemental Material Ref. [26]. The transition temperature $T_{\mathrm{c}}$ has been determined as the temperature where $\chi(T)$ has dropped by $10 \%$ of the normalised step, i.e., close to the onset of the transition. This procedure results in uncertainty less than $0.05 \mathrm{~K}$ of $T_{\mathrm{c}}$.

\section{EXPERIMENTAL RESULTS}

Our high-pressure Hall effect measurements show the suppression of $T_{\mathrm{CDW}}$ under pressure in Fig. 1(a). At high temperatures, the Hall coefficient, $R_{\mathrm{H}}$, is weakly temperature dependent and does not change with pressure indicating that the electronic structure in the non-CDW state remains unchanged by pressure. At $T_{\mathrm{CDW}}, R_{\mathrm{H}}(T)$ shows a large drop and a sign change consistent with earlier results at ambient and low pressure [27-29]. Such a sign change has been linked to the $\mathrm{CDW}$ transition in a variety of systems including $2 \mathrm{H}-\mathrm{NbSe}_{2}[14,24]$, cuprate $\mathrm{YBa}_{2} \mathrm{Cu}_{3} \mathrm{O}_{y}$ [30], and $1 \mathrm{~T}-\mathrm{TiSe}_{2}$ [31] and has been confirmed by model calculations [32]. The contribution of the CDW to the Hall coefficient $\Delta R_{\mathrm{H}}(T, P)=$ $R_{\mathrm{H}}(T, P)-R_{\mathrm{H}}(T, 5.5 \mathrm{GPa})$, is calculated by subtracting the non-CDW form well above the critical pressure. In the derivative, $d \Delta R_{\mathrm{H}} / d T$, the CDW transition manifests as a pronounced peak as shown in Fig. 1(c). $T_{\mathrm{CDW}}(P)$ associated with the maximum in $d \Delta R_{\mathrm{H}} / d T$ shifts to lower temperature as pressure is increased in good agreement with $T_{\mathrm{CDW}}(P)$ extracted from resistivity measurements as well as with previous results of $T_{\mathrm{CDW}}$ as highlighted in Fig. 2(a). The benefit of analyzing the Hall coefficient is that the strong signature can be traced to higher pressures where the signature in resistivity is lost [33]. We observe the CDW transition in $\Delta R_{\mathrm{H}}(T)$ up to a pressure of $4.3 \mathrm{GPa}$.

The CDW transition temperature drops abruptly above 4.3(1) $\mathrm{GPa}$ as can be seen from both the isobaric temperature dependence and the isothermal pressure dependence of the Hall coefficient. The peak in the isobaric temperature dependence $d \Delta R_{\mathrm{H}} / d T$ is reduced in amplitude above $3 \mathrm{GPa}$ as shown in Fig. 1(d). In fact, the reduction is most consistent with a power-law suppression where the amplitude vanishes at 4.3(1) GPa suggesting an absence of the CDW above this pressure. Thus we conclude that the CDW signature is absent from the temperature dependence of the Hall effect and highlight this in the phase diagram as $T_{\mathrm{CDW}}(4.4 \mathrm{GPa})=0$ [blue triangle at $4.4 \mathrm{GPa}$ in Fig. 2(a)]. The isothermal pressure dependence $R_{\mathrm{H}}(P)$ exhibits a pronounced kink associated with the critical pressure of the CDW phase, $P_{\mathrm{CDW}}(T)$ [cf. intersecting linear fits in Fig. 1(b)]. The position of $P_{\mathrm{CDW}}(T)$ is included as black squares in the phase diagram in Fig. 2(a). $P_{\mathrm{CDW}}(T)$ becomes independent of temperature for $T \leqslant 10 \mathrm{~K}$, i.e., the kink in $R_{\mathrm{H}}(P)$ is found at the same pressure $P_{\mathrm{CDW}}(T)=4.4 \mathrm{GPa}$ for 5 and $10 \mathrm{~K}$. In Sec. S I of the Supplemental Material Ref. [26], we show that this result is also true if the Hall effect is probed in smaller magnetic fields.

Superconductivity is boosted under pressure in clear anticorrelation to the CDW. We trace $T_{\mathrm{c}}(P)$ as the onset of the diamagnetic signal in magnetic susceptibility measure- 

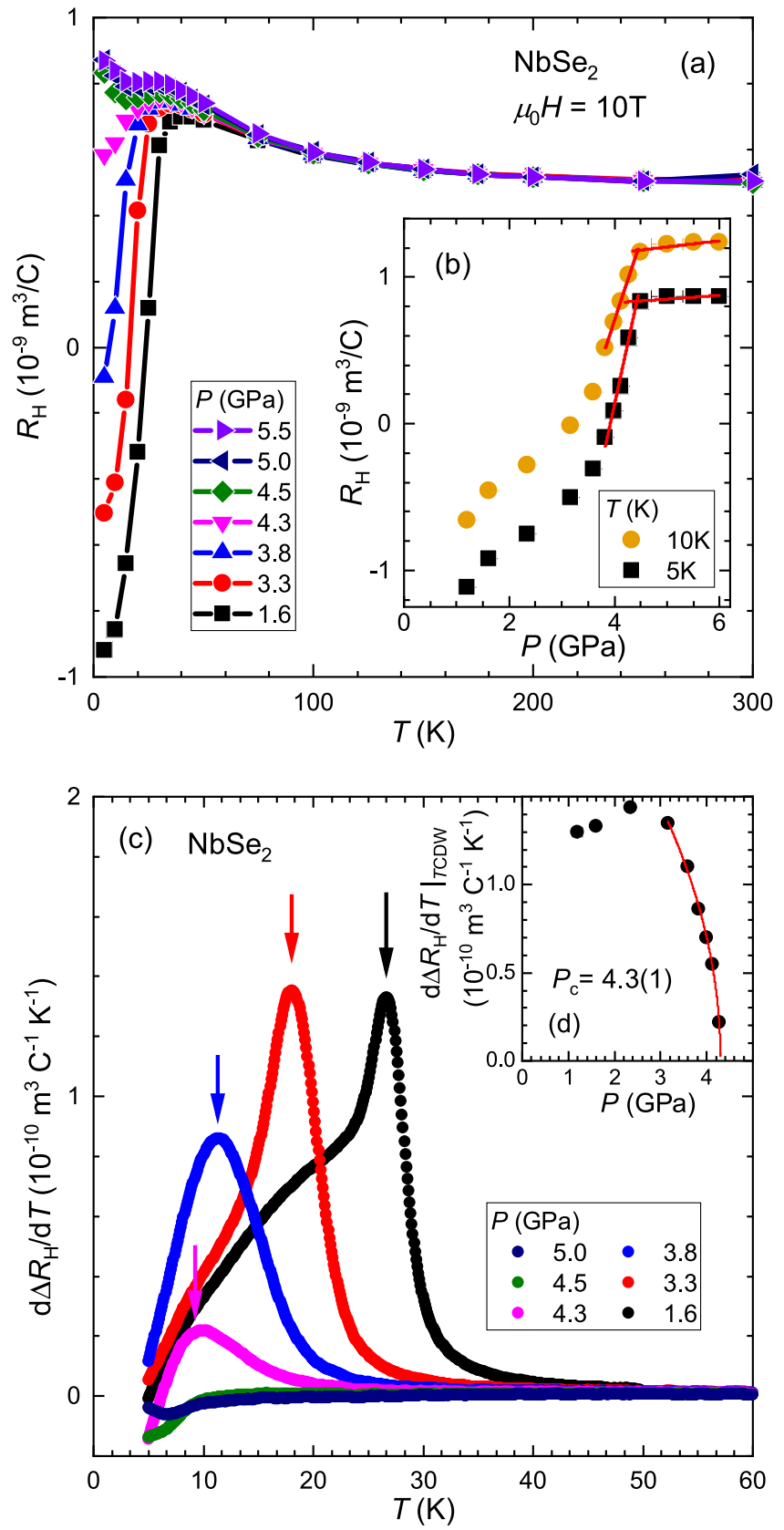

FIG. 1. Suppression of the CDW transition in $2 \mathrm{H}-\mathrm{NbSe}_{2}$ under pressure. (a) The temperature dependence of the Hall coefficient for sample 1. (b) Pressure dependence $R_{\mathrm{H}}(P)$ measured at $\mu_{0} H=$ $10 \mathrm{~T}$ at selected temperatures. Straight lines highlight linear fits to the data. (c) Derivative of the Hall coefficient $\left(d \Delta R_{\mathrm{H}} / d T\right)$ was calculated after subtracting the high-pressure background, i.e., $\Delta R_{\mathrm{H}}(T, P)=R_{\mathrm{H}}(T, P)-R_{\mathrm{H}}(T, 5.5 \mathrm{GPa})$. Arrows indicate $T_{\mathrm{CDW}}$ extracted as the maximum. (d) Amplitude of the peak in $d R_{\mathrm{H}} / d T$. Solid line denotes empirical power-law fit $d \Delta R_{\mathrm{H}} /\left.d T\right|_{T_{\mathrm{CDW}}}=$ $A\left|P-P_{\mathrm{c}}\right|^{b}$ to the data above $3 \mathrm{GPa}$ giving a critical pressure $P_{\mathrm{c}}=$ 4.3(1) GPa.

ments, $\chi(T)$, as presented in Fig. 3(a). The sharp onset gives $T_{\mathrm{c}}=7.1 \mathrm{~K}$ at ambient pressure in good agreement with our resistivity measurements (cf. S II of the Supplemental Material Ref. [26]) and other published work, e.g., Refs. [16,17,34]. With increasing pressure, $T_{\mathrm{c}}(P)$ shifts to higher temperature,
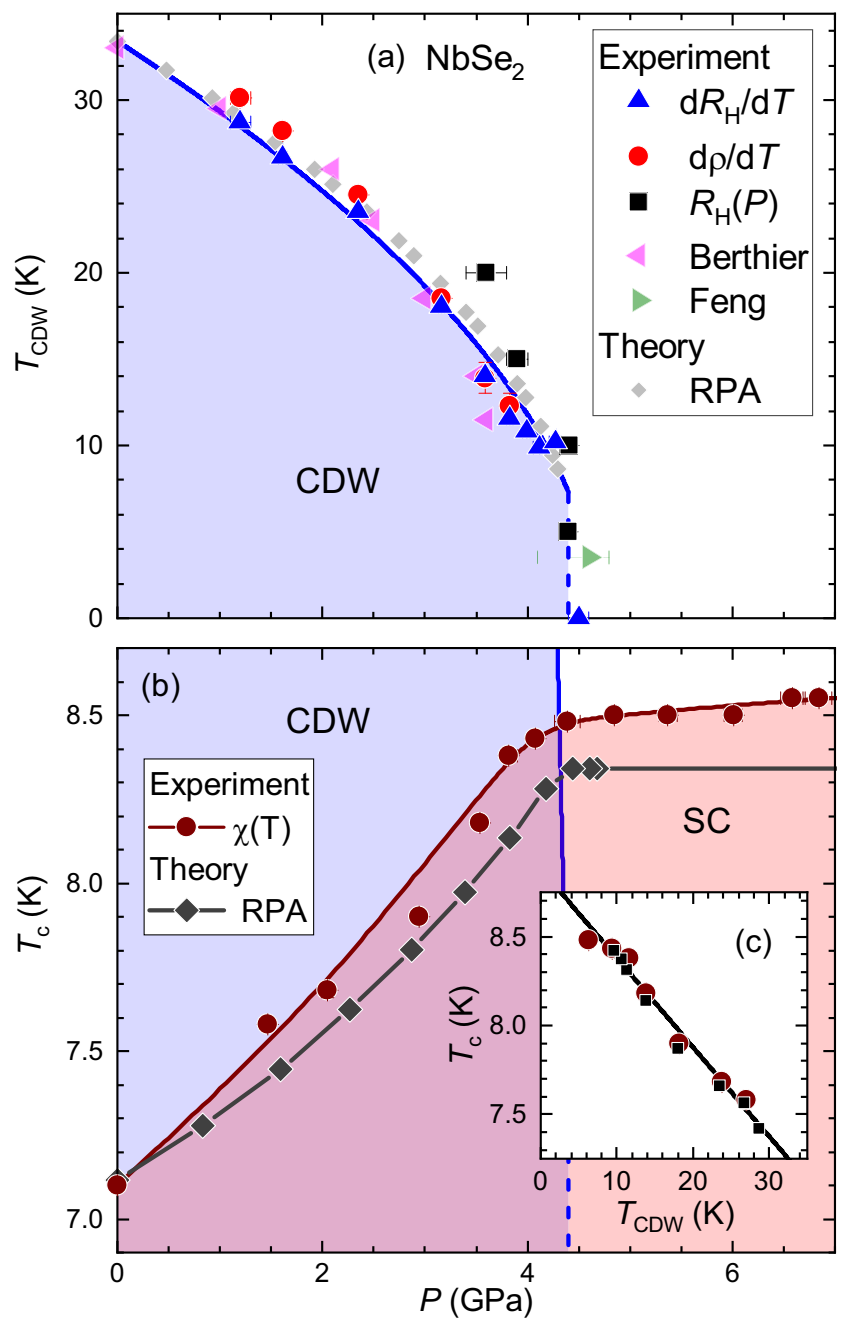

FIG. 2. High-pressure phase diagram of $2 \mathrm{H}-\mathrm{NbSe}_{2}$. (a) Experimental values of $T_{\mathrm{CDW}}(P)$ are determined as the peak in $d \Delta R_{\mathrm{H}} / d T$ as shown in Fig. 1, as the minimum in $d \rho / d T$ as shown in SI, and the kink in $R_{\mathrm{H}}(P)$ as shown Fig. 1(b). Theoretical values of $T_{\mathrm{CDW}}(P)$ are calculated as described in section $\mathrm{S} \mathrm{V}$ and $\mathrm{S}$ VI of the Supplemental Material Ref. [26]. Data from Refs. [22,33] are included. The solid line marks a power-law fit to our experimental datasets of $T_{\mathrm{CDW}}(P)$ for $P \leqslant 4.3 \mathrm{GPa}$. The dashed line illustrates the sharp decrease of $T_{\mathrm{CDW}}$ at $4.4 \mathrm{GPa}$ (see text). (b) Experimental results for $T_{\mathrm{c}}(P)$ are extracted from magnetization measurements [Fig. 3(a)]. A detailed comparison of $T_{\mathrm{c}}(P)$ using different pressure media is given in $\mathrm{S}$ I. Theoretical values of $T_{\mathrm{c}}(P)$ are calculated as described in $\mathrm{S}$ VI. The boundary of the CDW phase is reproduced from (a). Inset (c) shows the relation of the superconductivity and CDW transition temperatures for two samples: Red circles and black squares denote $T_{\mathrm{c}}$ of sample 1 and 2 detected with $\chi(T)$ and $\rho(T)$, respectively. The solid line is a linear fit.

while the transition remains very sharp. Above $4.4 \mathrm{GPa}, T_{\mathrm{c}}(P)$ saturates at $8.5 \mathrm{~K}$. The measurements presented in Fig. 3(a) used argon as a pressure transmitting medium (PTM) which remains hydrostatic up to $11 \mathrm{GPa}$ [35]. We find very good agreement with $T_{\mathrm{c}}(P)$ extracted from our resistivity measurements up to $5.5 \mathrm{GPa}$-the limiting pressure for hydrostaticity of the PTM glycerol used for the electrical transport measurements [35]. 

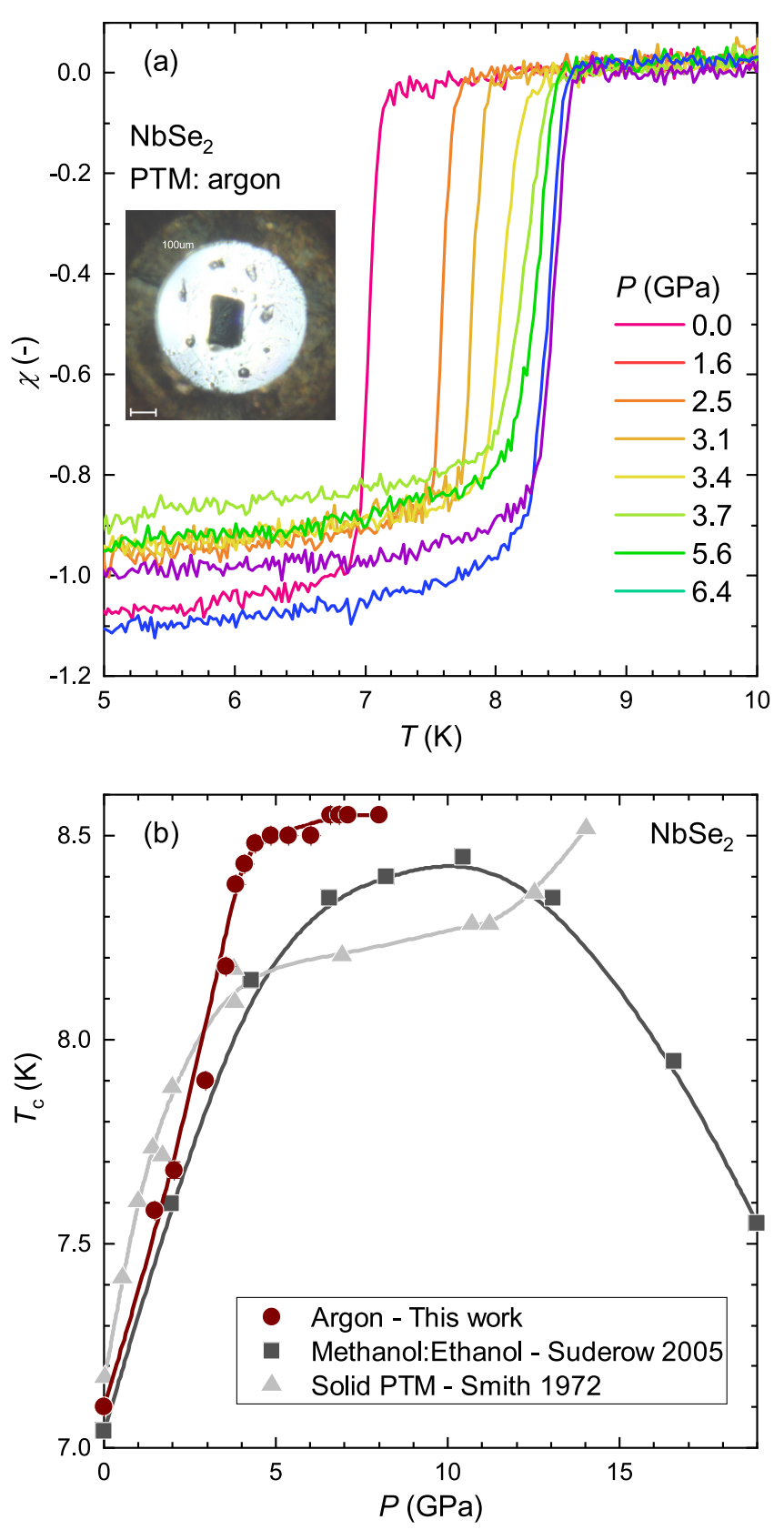

FIG. 3. Enhanced superconductivity in $2 \mathrm{H}-\mathrm{NbSe}_{2}$ under pressure. (a) The volume susceptibility $\chi$ measured on warming for sample 2 in a magnetic field $\mu_{0} H=0.5 \mathrm{mT}$ after zero-field cooling. The transition into the superconducting state inferred from the diamagnetic signal shifts to higher temperatures as the pressure is increased. Inset shows a picture of the sample and ruby chips inside the pressure cell. Argon was used as a pressure medium. (b) Comparison of our measurements with previous studies beyond the CDW critical pressure $[23,36]$.

Our measurements of $T_{\mathrm{c}}(P)$ differ significantly from the two previous studies which extend beyond the critical pressure of the CDW by Smith et al. [36] and Suderow et al. [23] as presented in Fig. 3(b). A similar initial slope of $T_{\mathrm{c}}(P)$ is seen in our work and those previous studies (including further work limited to below $3 \mathrm{GPa}[33,34])$. However, a marked difference is observed above $4 \mathrm{GPa}$ where all datasets show a plateau at different values of $T_{\mathrm{c}}$. Notably, we observe the highest $T_{\mathrm{c}}$ in any of the measurements. Given that impurities have been shown to cause a suppression of superconductivity outside the CDW phase [24,25], this suggests that the studies by Smith et al. and Suderow et al. suffered from sample impurities or inhomogeneities. Our electrical resistivity measurements reveal that the residual resistance ratio remains large at above 60 while Smith et al. and Suderow et al. have not provided a characterization of their samples at ambient pressure and could not monitor the pressure inhomogeneity effects with the ac susceptibility measurements. In fact, Smith et al. used non-hydrostatic solid pressure medium, which is know to lead to pressure inhomogeneities and anisotropy. In the case of Suderow et al., methanol:ethanol was used with a hydrostatic limit of $\approx 9 \mathrm{GPa}$ [35]. However, we show in the S II of the Supplemental Material Ref. [26] that the sample preparation and stresses from the sample touching the gasket can lead to a reduced $T_{\mathrm{c}}$ outside the CDW phase and we reproduce the $T_{\mathrm{c}}(P)$ of Suderow in a sample with a broadened transition. In summary, we argue that our data for the first time reveal the intrinsic high-pressure evolution of the superconducting transition temperature of $2 \mathrm{H}-\mathrm{NbSe}_{2}$.

We use measurements of the upper critical field $H_{\mathrm{c} 2}$ to characterize the Fermi velocity, $v_{\mathrm{F}}$, on the strong coupling $\mathrm{Nb}$ cylinders. At all pressures, we observe a linear dependency of $H_{\mathrm{c} 2}(T)$ below $T_{\mathrm{c}}$ as presented in Fig. 4(a) and in agreement with earlier ambient-pressure experiments [24,37]. We use the slope $d H_{\mathrm{c} 2} /\left.d T\right|_{T_{\mathrm{c}}}$ to calculate the Fermi velocity $v_{\mathrm{F}}$, shown in Fig. 4(b). Both ab initio calculations and analysis of the Usadel equations show that $d H_{\mathrm{c} 2} /\left.d T\right|_{T_{\mathrm{c}}}$ for $H$ along the crystallographic $c$ direction, used here, is almost entirely determined by the strong coupling $\mathrm{Nb}$ cylinders [38,39]. In addition, we find perfect agreement with $v_{\mathrm{F}}$ associated with the $\mathrm{Nb}$ cylinders identified in the previous high-pressure measurements by Suderow et al. at low pressures in the pressure range where $T_{\mathrm{c}}$ of Suderow's sample is in agreement with our measurements [23] [grey squares in Fig. 4(b)].

At the critical pressure of the CDW phase, we observe a jump in the Fermi velocity suggesting a collapse of the CDW gap [dotted line in Fig. 4(b)]. Initially, a steady increase of $v_{\mathrm{F}}$ by $40 \%$ is observed for $P \leqslant 4.3 \mathrm{GPa}$, i.e., where $T_{\mathrm{c}}<T_{\mathrm{CDW}}$. This initial steady rise is most naturally associated with a continuous shrinkage of the CDW gap and reduction of the average renormalization on the niobium bands. By contrast, a jump of $\approx 10 \%$ is observed over a narrow pressure range at $P \sim 4.4 \mathrm{GPa}$, exactly at the pressure where $T_{\mathrm{CDW}}$ drops to zero consistent with a collapse of the CDW gap. Above $5 \mathrm{GPa} v_{\mathrm{F}}$ saturates suggesting that the coupling of the CDW mode to the electronic states quickly reduces outside the CDW phase. As $H_{\mathrm{c} 2}(T)$ is well fitted by a linear dependence to lowest magnetic fields (i.e., right to $T_{\mathrm{c}}$ ), we conclude that the CDW collapse is also present in zero magnetic field at 4.4 GPa corroborating the evidence for a drop in $T_{\mathrm{CDW}}$ from the Hall-effect measurements in magnetic fields above $2 \mathrm{~T}$ discussed above.

\section{THEORETICAL MODELLING}

We find that the stiffening of the bare longitudinal acoustic phonon from which the CDW develops can account for the suppression of $T_{\mathrm{CDW}}$ under pressure. We use electron-phonon 

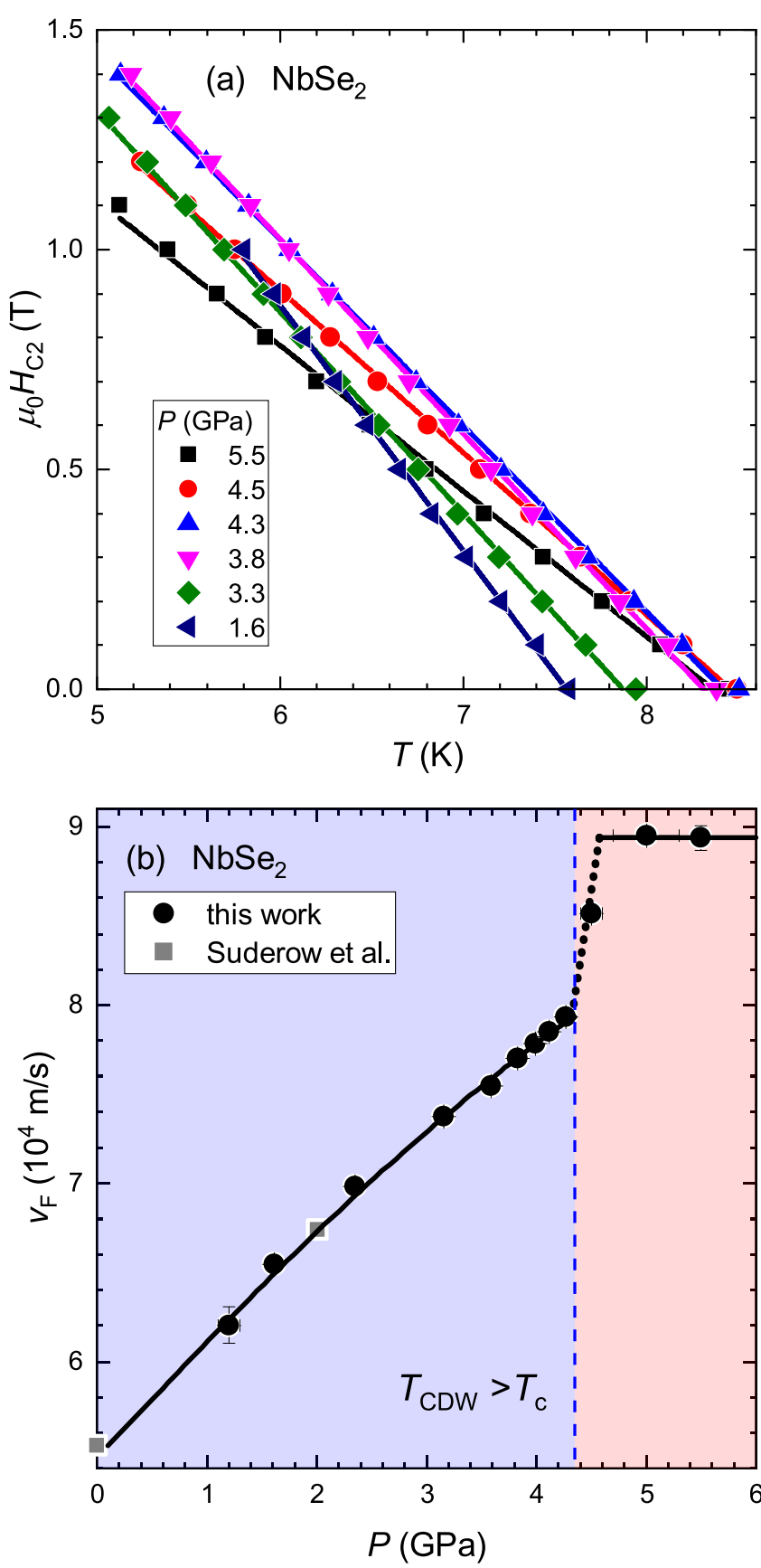

FIG. 4. Upper critical field and Fermi velocity in $2 \mathrm{H}-\mathrm{NbSe}_{2}$. (a) Linear fits to $H_{\mathrm{c} 2}(T)$ have been used to extract $v_{\mathrm{F}}(P)$ shown in (b). For details see S III of the Supplemental Material Ref. [26]. Data of Suderow et al. [23] have been included in (b) for the pressure range with good agreement of $T_{\mathrm{c}}$ (see SII of the Supplemental Material Ref. [26]). Lines are guides to the eye.

coupling dependent on the ingoing and outgoing momentum and the specific shape of the Fermi surface of $2 \mathrm{H}-\mathrm{NbSe}_{2}$ including the orbital character. The model was developed earlier by one of us as outlined in (Secs. S V and S VI of the Supplemental Material Ref. [26]) [40,41]. In our random phase approximation (RPA) calculations, the overall magnitude of the electron-phonon coupling $\boldsymbol{g}$ is constrained to reproduce $T_{\mathrm{CDW}}(P=0)=33.4 \mathrm{~K}$ (cf. Sec. S III of the Supplemental Material Ref. [26]) and we keep $g$ fixed for all

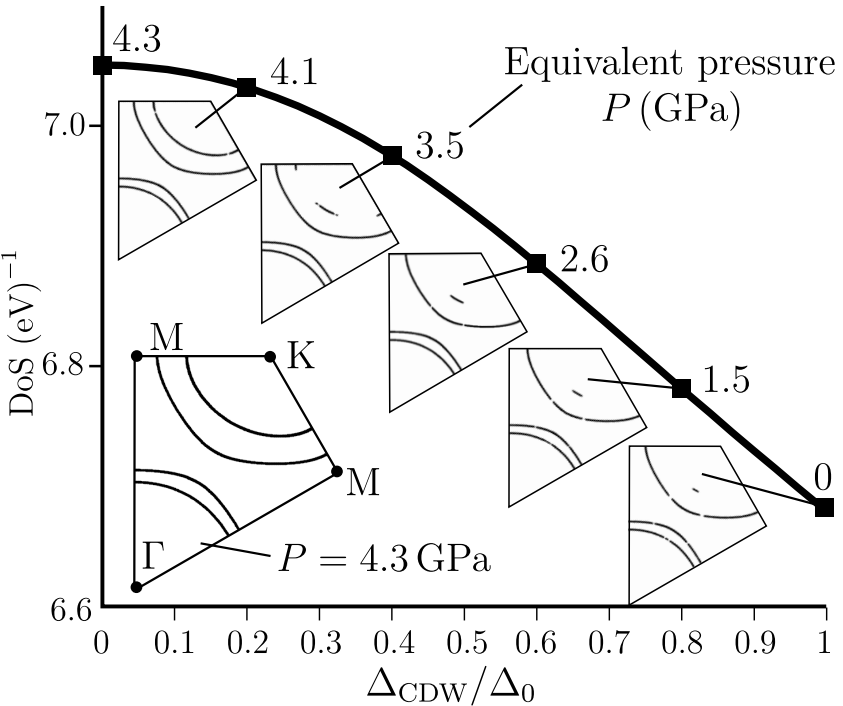

FIG. 5. Reduction of DOS as a function of CDW gap magnitude for the two $\mathrm{Nb}$-derived bands at $E_{\mathrm{F}}$. Insets show the Fermi surface in a wedge of the Brillouin zone for specific values of $\Delta_{\mathrm{CDW}}$ and equivalent pressure. The plotted points were identified as the points at which the RPA spectral function is within $15 \%$ of its maximum value.

pressures. To describe $T_{\mathrm{CDW}}(P)$, we assume a linear stiffening of the longitudinal acoustic phonons underlying the CDW formation consistent with high-pressure inelastic X-ray studies [42] as detailed in section S V of the Supplemental Material Ref. [26]. In Fig. 2(a), the experimental transition temperatures are compared to model calculations. From the good match with the experimental phase boundary up to $4.3 \mathrm{GPa}$, we conclude that the suppression of the CDW is indeed driven by the increase of the bare phonon frequency, while the electron-phonon constant remains unchanged. While our model tracks the phase boundary well for $P<4.3 \mathrm{GPa}$, it is too simple to account for a possible change in the order of the transition. Features omitted from the model which could account for such a change include higher-order lattice coupling, fluctuation effects, or the effect of pressure on the electronic band structure.

A partial competition for density of states (DOS) is the main driver for the evolution of $T_{\mathrm{c}}(P)$. We use the experimentally determined phase boundary [solid line in Fig. 2(a)] to scale the evolution of the CDW phase to our pressure data as detailed in Sec. S VI of the Supplemental Material Ref. [26]. Inside the CDW phase, the DOS available for superconductivity is reduced due to the gapping of the inner $K$ pockets of the Fermi surface as illustrated in Fig. 5 leading to a reduction of $T_{\mathrm{c}}$. As the CDW gap becomes smaller, the DOS available for superconductivity becomes larger which in turn accounts for almost the entire increase of $T_{\mathrm{c}}$ and naturally explains why $T_{\mathrm{c}}$ saturates above $P_{\mathrm{CDW}}$ as can be seen in Fig. 2(b). Thus we conclude that it is a competition for DOS which suppresses $T_{\mathrm{c}}$ inside the CDW phase.

\section{DISCUSSION AND CONCLUSIONS}

Our study provides the most comprehensive and consistent dataset of the boundary of the CDW phase in $2 \mathrm{H}-\mathrm{NbSe}_{2}$ to 
date and suggests a first-order transition at $P_{\mathrm{CDW}}=4.4 \mathrm{GPa}$. The combined evidence of a drop in $T_{\mathrm{CDW}}(P)$ at $P_{\mathrm{CDW}}$ extracted from the isobaric and isothermal analysis of the Hall effect and the jump in $v_{\mathrm{F}}(P)$ at $P_{\mathrm{CDW}}$ suggest that the first-order transition at $P_{\mathrm{CDW}}$ is an intrinsic characteristic of the $\mathrm{CDW}$ in $2 \mathrm{H}-\mathrm{NbSe}_{2}$.

Our work is the first high-pressure study to find indications of a first-order transition at the critical pressure of the CDW order. The previous pressure study by Berthier et al. traced $T_{\mathrm{CDW}}$ to $3.6 \mathrm{GPa}$ [33]. Only one further study has obtained information about the boundary of the CDW phase above 3.7 GPa: the X-ray measurements of Feng et al. [22,43] who have interpreted a kink in the pressure dependence of the $c$-axis lattice constant at $4.6 \mathrm{GPa}$ and $3.5 \mathrm{~K}$ as the critical pressure of the CDW phase [green triangle in Fig. 2(a)]. An uncertainty has not been given by Feng et al., however, taking into account the scatter of $c(P)$ we estimate an uncertainty of $4.6(-0.5)(+0.2) \mathrm{GPa}$ as included in our phase diagram Fig. 2(a). Taking into account this uncertainty, the data of Feng et al. are consistent with the critical pressure of $4.4 \mathrm{GPa}$ found in our study.

The XRD measurements of Feng et al. cannot rule out a first-order transition at $4.4 \mathrm{GPa}[22,43]$. The in-plane lattice constant $a$ was measured with very high precision up to $8.5 \mathrm{GPa}$ but does not reveal any signature at $P_{\mathrm{CDW}}$ and thus cannot be used to discriminate the order of the quantum phase transition. Together the lattice constants give an upper limit of $0.5 \%$ for a discontinuity in the volume leaving the possibility of a weak first-order transition compatible with the small $T_{\mathrm{CDW}} \approx 10 \mathrm{~K}$ just below $P_{\mathrm{CDW}}$. Scaling in the $\mathrm{x}$-ray diffraction measurements of Feng et al. is cut off above $4 \mathrm{GPa}$ $[22,43]$. At $4.55 \mathrm{GPa}$ the XRD measurements find a cut-off of the divergence of the inverse static correlation length and an order-of magnitude drop of the CDW intensity. Only the low-pressure XRD data show clear evidence of second-order behavior (divergence of the inverse static correlation length and smooth decrease of the intensity of the CDW reflections up to $4 \mathrm{GPa}$ ) consistent with our continuous suppression of $T_{\mathrm{CDW}}(P)$ up to $4.3 \mathrm{GPa}$.

Indications of a first-order transition were observed before in electron-irradiated $2 \mathrm{H}-\mathrm{NbSe}_{2}$ but were attributed to disorder effects [25]. Our samples preserve the high residual resistance ratio across $P_{\mathrm{CDW}}$ thus disorder is of negligible effect in our study. Instead, our results suggest that quantum fluctuations or coupling to the lattice may induce a first-order transition in $2 \mathrm{H}-\mathrm{NbSe}_{2}$. Indeed, a suppression of CDW order by quantum fluctuations was proposed for $2 \mathrm{H}-\mathrm{NbSe}_{2}[42,43]$ and a strong coupling to the lattice plays a crucial part for the formation of the CDW in $2 \mathrm{H}-\mathrm{NbSe}_{2}$ [44]. A weak first-order phase transition to the incommensurate CDW state was observed in related CDW systems $1 T-\mathrm{TiSe}_{2}$ and $1 T-\mathrm{TaS}_{2}[14,45]$. Thus our results suggest that quantum fluctuations and/or coupling to the lattice play an important role when suppressing CDW order to zero temperature and induce a first-order transition in many CDW materials.

Our detailed measurements lead us to different conclusions on the interplay between superconductivity and CDW order compared to previous studies. The increase in $T_{\mathrm{c}}$ is in clear anticorrelation with $T_{\mathrm{CDW}}(P)$ as highlighted in Fig. 2(c). In addition, $T_{\mathrm{c}}(P)$ is virtually constant for $P \geqslant 4.4 \mathrm{GPa}$, i.e. outside the CDW phase. This anticorrelation and the saturation are clear signs that the superconductivity is in competition with the CDW phase as suggested for CDW superconductors in general and $2 \mathrm{H}-\mathrm{NbSe}_{2}$ in particular in previous studies $[9,33]$. Our model calculations show quantitatively that the suppression is caused by the depletion of density of states inside the CDW phase. Notably, we do not observe a maximum in $T_{\mathrm{c}}$ around the critical pressure of the CDW phase. Thus we can rule out a boost to $T_{\mathrm{c}}$ from quantum critical fluctuations. Similarly, we can rule out a boost to superconductivity from the presence of the static CDW as suggested by Kiss et al. [12]. Such a boost should manifest in a correlation of $T_{\mathrm{CDW}}$ and $T_{\mathrm{c}}$ and a drop of $T_{\mathrm{c}}$ at $P_{\mathrm{CDW}}$ neither of which is observed. Furthermore, our data suggest that a notable maximum is absent for pressures below $10 \mathrm{GPa}$ thus suggesting that the electron-phonon coupling responsible for the superconductivity is largely independent of pressure.

The first-order nature of the $\mathrm{CDW}$ close to $P_{\mathrm{CDW}}$ maybe the reason for the absence of a dome-shaped enhancement in $T_{\mathrm{c}}(P)$ upon suppression of the CDW. At a second-order quantum phase transition, i.e., a quantum critical point, a superconducting dome was observed in many systems including at the CDW QCP in $1 \mathrm{~T}-\mathrm{TiSe}_{2}$ [10], close to the structural QCP in $(\mathrm{Sr}, \mathrm{Ca})_{3} \mathrm{Ir}_{4} \mathrm{Sn}_{13}$ [46], and at the antiferromagnetic QCP in $\mathrm{CePd}_{2} \mathrm{Si}_{2}$ [5]. By contrast, dome-shaped superconducting phases are usually absent in systems with first-order quantum phase transitions as shown for instance in $\mathrm{UGe}_{2}$ [47]. This is a clear indication that the gapped fluctuation spectrum at first-order quantum phase transitions is not suitable to mediate or enhance superconductivity. Our data suggest that this principle applies to $2 \mathrm{H}-\mathrm{NbSe}_{2}$, too.

While we have shown clearly that CDW order is suppressing superconductivity, our data also reveal that superconductivity has no effect on $T_{\mathrm{CDW}}$. Firstly, the gradual suppression of $T_{\mathrm{CDW}}$ for $P<4 \mathrm{GPa}$ cannot be driven by the superconductivity because $T_{\mathrm{CDW}}>T_{\mathrm{c}}$. Secondly, while the drop of $T_{\mathrm{CDW}}$ occurs at the pressure where the power-law fit predicts $T_{\mathrm{CDW}}<T_{\mathrm{c}}(H=0)$, our Hall-effect data have detected the CDW transition in high magnetic fields where superconductivity is suppressed. In addition, the suppression of the amplitude (peak height) of the CDW signature in $R_{\mathrm{H}}(T)$ is based entirely on data where $T_{\mathrm{CDW}}>T_{\mathrm{c}}(H=0)$. Thus our data suggest that the drop of $T_{\mathrm{CDW}}$ at $4.4 \mathrm{GPa}$ is intrinsic to the $\mathrm{CDW}$ in $2 \mathrm{H}-\mathrm{NbSe}_{2}$ and not driven by competition with superconductivity. Hence, we conclude that the competition between superconductivity and CDW is mostly unidirectional in $2 \mathrm{H}-\mathrm{NbSe}_{2}$ with only superconductivity suppressed by CDW but not the other way around. Such a unidirectional competition is supported by previous $\mathrm{X}$-ray measurements at ambient pressure, which show that the intensity of the CDW reflection is not reduced at $T_{\mathrm{c}}$ in zero field and not enhanced upon suppressing superconductivity in large magnetic fields $[14,48]$.

In summary, our results lead to several profound conclusions about the interplay of CDW order and superconductivity in $2 \mathrm{H}-\mathrm{NbSe}_{2}$ : (i) Superconductivity is suppressed inside the CDW phase due the reduced electronic density 
of states available for superconductivity, (ii) Superconductivity is not reducing $T_{\mathrm{CDW}}$. Instead the suppression of $T_{\mathrm{CDW}}$ under pressure is consistent with the stiffening of the underlying bare phonon mode. (iii) $T_{\mathrm{CDW}}$ drops abruptly at $P_{\mathrm{CDW}}=4.4 \mathrm{GPa}$ indicating a first-order transition. (iv) Superconductivity is not enhanced at $P_{\mathrm{CDW}}$ potentially due to CDW fluctuations being cut off at the first-order transition.

Data are available at the University of Bristol data repository, see Ref. [49].

\section{ACKNOWLEDGMENTS}

The authors would like to thank Jasper van Wezel, Jans Henke, Nigel Hussey, Hermann Suderow, and Antony Carrington for valuable discussion. The authors acknowledge supported by the EPSRC under Grants No. EP/L015544/1, No. EP/R011141/1, No. EP/L025736/1, and No. EP/N026691/1 as well as the ERC Horizon 2020 programme under Grant No. 715262-HPSuper. F.F. acknowledges support from the Astor Junior Research Fellowship of New College, Oxford.
[1] M. Nakano, K. Shibuya, D. Okuyama, T. Hatano, S. Ono, M. Kawasaki, Y. Iwasa, and Y. Tokura, Collective bulk carrier delocalization driven by electrostatic surface charge accumulation, Nature (London) 487, 459 (2012).

[2] B. Baek, W. H. Rippard, S. P. Benz, S. E. Russek, and P. D. Dresselhaus, Hybrid superconducting-magnetic memory device using competing order parameters, Nat. Commun. 5, 3888 (2014).

[3] S. Friedemann, W. J. Duncan, M. Hirschberger, T. W. Bauer, R. Küchler, A. Neubauer, M. Brando, C. Pfleiderer, and F. M. Grosche, Quantum tricritical points in $\mathrm{NbFe}_{2}$, Nat. Phys. 14, 62 (2018).

[4] J. Chang, E. Blackburn, A. T. Holmes, N. B. Christensen, J. Larsen, J. Mesot, R. Liang, D. A. Bonn, W. N. Hardy, A. Watenphul, M. v. Zimmermann, E. M. Forgan, and S. M. Hayden, Direct observation of competition between superconductivity and charge density wave order in $\mathrm{YBa}_{2} \mathrm{Cu}_{3} \mathrm{O}_{6.67}$, Nat. Phys. 8, 871 (2012).

[5] N. D. Mathur, F. M. Grosche, S. R. Julian, I. R. Walker, D. M. Freye, R. K. W. Haselwimmer, and G. G. Lonzarich, Magnetically mediated superconductivity in heavy fermion compounds, Nature (London) 394, 39 (1998).

[6] M. Brando, D. Belitz, F. M. Grosche, and T. R. Kirkpatrick, Metallic quantum ferromagnets, Rev. Mod. Phys. 88, 025006 (2016).

[7] H. v. Löhneysen, A. Rosch, M. Vojta, and P. Wölfle, Fermiliquid instabilities at magnetic quantum phase transitions, Rev. Mod. Phys. 79, 1015 (2007).

[8] A. Frano, S. Blanco-Canosa, B. Keimer, and R. J. Birgeneau, Charge ordering in superconducting copper oxides, J. Phys.: Condens. Matter 32, 374005 (2020).

[9] G. Bilbro and W. McMillan, Theoretical model of superconductivity and the martensitic transformation in A15 compounds, Phys. Rev. B 14, 1887 (1976).

[10] E. Morosan, H. W. Zandbergen, B. S. Dennis, J. W. G. Bos, Y. Onose, T. Klimczuk, A. P. Ramirez, N. P. Ong, and R. J. Cava, Superconductivity in $\mathrm{Cu}_{x} \mathrm{TiSe}_{2}$, Nat. Phys. 2, 544 (2006).

[11] B. J. Ramshaw, S. E. Sebastian, R. D. McDonald, J. Day, B. S. Tan, Z. Zhu, J. B. Betts, R. Liang, D. A. Bonn, W. N. Hardy, and N. Harrison, Quasiparticle mass enhancement approaching optimal doping in a high- $\mathrm{T}_{c}$ superconductor, Science 348, 317 (2015).

[12] T. Kiss, T. Yokoya, A. Chainani, S. Shin, T. Hanaguri, M. Nohara, and H. Takagi, Charge-order-maximized momentum-dependent superconductivity, Nat. Phys. 3, 720 (2007).
[13] S. V. Borisenko, A. A. Kordyuk, V. B. Zabolotnyy, D. S. Inosov, D. Evtushinsky, B. Büchner, A. N. Yaresko, A. Varykhalov, R. Follath, W. Eberhardt, L. Patthey, and H. Berger, Two Energy Gaps and Fermi-Surface "Arcs" in $\mathrm{NbSe}_{2}$, Phys. Rev. Lett. 102 166402 (2009).

[14] D. E. Moncton, J. D. Axe, and F. J. DiSalvo, Study of Superlattice Formation in $2 \mathrm{H}-\mathrm{NbSe}_{2}$ and $2 \mathrm{H}-\mathrm{TaSe}_{2}$ by Neutron Scattering, Phys. Rev. Lett. 34, 734 (1975).

[15] T. Yokoya, T. Kiss, A. Chainani, S. Shin, M. Nohara, and H. Takagi, Fermi surface sheet-dependent superconductivity in $2 \mathrm{H}-\mathrm{NbSe}_{2}$, Science 294, 2518 (2001).

[16] J. A. Wilson and A. Yoffe, The transition metal dichalcogenides discussion and interpretation of the observed optical, electrical and structural properties, Adv. Phys. 18, 193 (1969).

[17] A. Wieteska, B. Foutty, Z. Guguchia, F. Flicker, B. Mazel, L. Fu, S. Jia, C. Marianetti, J. van Wezel, and A. Pasupathy, Uniaxial strain tuning of superconductivity in $2 \mathrm{H}-\mathrm{NbSe}_{2}$, arXiv:1903.05253.

[18] D. J. Rahn, S. Hellmann, M. Kalläne, C. Sohrt, T. K. Kim, L. Kipp, and K. Rossnagel, Gaps and kinks in the electronic structure of the superconductor $2 \mathrm{H}-\mathrm{NbSe}_{2}$ from angle-resolved photoemission at $1 \mathrm{k}$, Phys. Rev. B 85, 224532 (2012).

[19] T. Valla, A. V. Fedorov, P. D. Johnson, P.-A. Glans, C. McGuinness, K. E. Smith, E. Y. Andrei, and H. Berger, Quasiparticle Spectra, Charge-Density Waves, Superconductivity, and Electron-Phonon Coupling in $2 \mathrm{H}-\mathrm{NbSe}_{2}$, Phys. Rev. Lett. 92, 086401 (2004)

[20] J. D. Fletcher, A. Carrington, P. Diener, P. Rodière, J. P. Brison, R. Prozorov, T. Olheiser, and R. W. Giannetta, Penetration Depth Study of Superconducting Gap Structure of $2 \mathrm{H}-\mathrm{NbSe}_{2}$, Phys. Rev. Lett. 98, 057003 (2007).

[21] J. A. Galvis, E. Herrera, C. Berthod, S. Vieira, I. Guillamón, and $\mathrm{H}$. Suderow, Tilted vortex cores and superconducting gap anisotropy in $2 \mathrm{H}-\mathrm{NbSe}_{2}$, Commun. Phys. 1, 30 (2018).

[22] Y. Feng, J. Wang, R. Jaramillo, J. van Wezel, S. Haravifard, G. Srajer, Y. Liu, Z.-A. Z.-A. Xu, P. B. Littlewood, and T. F. Rosenbaum, Order parameter fluctuations at a buried quantum critical point., Proc. Natl. Acad. Sci. USA 109, 7224 (2012).

[23] H. Suderow, V. G. Tissen, J. P. Brison, J. L. Martínez, and S. Vieira, Pressure Induced Effects on the Fermi Surface of Superconducting $2 \mathrm{H}-\mathrm{NbSe}_{2}$, Phys. Rev. Lett. 95, 117006 (2005).

[24] B. J. Dalrymple and D. E. Prober, Upper critical fields of the superconducting layered compounds $\mathrm{Nb}_{1-x} \mathrm{Ta}_{x} \mathrm{Se}_{2}$, J. Low Temp. Phys. 56, 545 (1984).

[25] K. Cho, M. Kończykowski, S. Teknowijoyo, M. A. Tanatar, J. Guss, P. B. Gartin, J. M. Wilde, A. Kreyssig, R. J. McQueeney, A. I. Goldman, V. Mishra, P. J. Hirschfeld, and R. Prozorov, 
Using controlled disorder to probe the interplay between charge order and superconductivity in $\mathrm{NbSe}_{2}$, Nat. Commun. 9, 2796 (2018).

[26] See Supplemental Material at http://link.aps.org/supplemental/ 10.1103/PhysRevResearch.2.043392 for details.

[27] H. N. S. Lee, M. Garcia, H. McKinzie, and A. Wold, The low-temperature electrical and magnetic properties of $\mathrm{TaSe}_{2}$ and $\mathrm{NbSe}_{2}$, J. Solid State Chem. 1, 190 (1970).

[28] K. Yamaya and T. Sambongi, Low-temperature crystal modification and the superconductive transition temperature of $\mathrm{NbSe}_{2}$, Solid State Commun. 11, 903 (1972).

[29] D. J. Huntley and R. F. Frindt, Transport properties of $\mathrm{NbSe}_{2}$, Can. J. Phys. 52, 861 (1973).

[30] D. LeBoeuf, N. Doiron-Leyraud, B. Vignolle, M. Sutherland, B. J. Ramshaw, J. Levallois, R. Daou, F. Laliberté, O. Cyr-Choinière, J. Chang, Y. J. Jo, L. Balicas, R. Liang, D. A. Bonn, W. N. Hardy, C. Proust, and L. Taillefer, Lifshitz critical point in the cuprate superconductor $\mathrm{Yba}_{2} \mathrm{Cu}_{3} \mathrm{O}_{y}$ from high-field Hall effect measurements, Phys. Rev. B 83, 054506 (2011).

[31] P. Knowles, B. Yang, T. Muramatsu, O. Moulding, J. Buhot, C. J. Sayers, E. DaComo, and S. Friedemann, Fermi Surface Reconstruction and Electron Dynamics at the Charge-DensityWave Transition in $\mathrm{TiSe}_{2}$, Phys. Rev. Lett. 124, 167602 (2020).

[32] D. V. Evtushinsky, A. A. Kordyuk, V. B. Zabolotnyy, D. S. Inosov, B. Buchner, H. Berger, L. Patthey, R. Follath, and S. V. Borisenko, Pseudogap-Driven Sign Reversal of the Hall Effect, Phys. Rev. Lett. 100, 236402 (2008).

[33] C. Berthier, P. Molinié, and D. Jérome, Evidence for a connection between charge density waves and the pressure enhancement of superconductivity in $2 \mathrm{H}-\mathrm{NbSe}_{2}$, Solid State Commun. 18, 1393 (1976).

[34] F. O. von Rohr, J.-C. Orain, R. Khasanov, C. Witteveen, Z. Shermadini, A. Nikitin, J. Chang, A. R. Wieteska, A. N. Pasupathy, M. Z. Hasan, A. Amato, H. Luetkens, Y. J. Uemura, and Z. Guguchia, Unconventional scaling of the superfluid density with the critical temperature in transition metal dichalcogenides, Sci. Adv. 5, eaav8465 (2019).

[35] N. Tateiwa and Y. Haga, Evaluations of pressure-transmitting media for cryogenic experiments with diamond anvil cell, Rev. Sci. Instrum. 80, 123901 (2009).

[36] T. F. Smith, L. E. Delong, A. R. Moodenbough, T. H. Geballe, and R. E. Schwall, Superconductivity of $\mathrm{NbSe}_{2}$ to $140 \mathrm{kbar}$, J. Phys. C 5, L230 (1972).

[37] N. Toyota, H. Nakatsuji, K. Noto, A. Hoshi, N. Kobayashi, Y. Muto, and Y. Onodera, Temperature and angular dependences of upper critical fields for the layer structure superconductor $2 \mathrm{H}-\mathrm{NbSe}_{2}$, J. Low Temp. Phys. 25, 485 (1976).
[38] M. Arai and T. Kita, Ab initio calculations of $H_{\mathrm{c} 2}$ for $\mathrm{Nb}, \mathrm{NbSe}_{2}$, and $\mathrm{MgB}_{2}$, J. Phys. Soc. Jpn. 73, 2924 (2004).

[39] A. A. Golubov and A. E. Koshelev, Upper critical field in dirty two-band superconductors: Breakdown of the anisotropic Ginzburg-Landau theory, Phys. Rev. B 68, 104503 (2003).

[40] F. Flicker and J. van Wezel, Charge order from orbitaldependent coupling evidenced by $\mathrm{NbSe}_{2}$, Nat. Commun. 6, 7034 (2015).

[41] F. Flicker and J. van Wezel, Charge order in $\mathrm{NbSe}_{2}$, Phys. Rev. B 94, 235135 (2016).

[42] M. Leroux, I. Errea, M. Le Tacon, S.-M. Souliou, G. Garbarino, L. Cario, A. Bosak, F. Mauri, M. Calandra, and P. Rodière, Strong anharmonicity induces quantum melting of charge density wave in $2 \mathrm{H}-\mathrm{NbSe}_{2}$ under pressure, Phys. Rev. B 92 , 140303(R) (2015).

[43] Y. Feng, J. van Wezel, J. Wang, F. Flicker, D. M. Silevitch, P. B. Littlewood, and T. F. Rosenbaum, Itinerant density wave instabilities at classical and quantum critical points, Nat. Phys. 11, 865 (2015).

[44] F. Weber, S. Rosenkranz, J. P. Castellan, R. Osborn, R. Hott, R. Heid, K.-P. Bohnen, T. Egami, A. H. Said, and D. Reznik, Extended Phonon Collapse and the Origin of the Charge-Density Wave in $2 H-\mathrm{NbSe}_{2}$, Phys. Rev. Lett. 107, 107403 (2011).

[45] Y. I. Joe, X. M. Chen, P. Ghaemi, K. D. Finkelstein, G. A. de la Pena, Y. Gan, J. C. T. Lee, S. Yuan, J. Geck, G. J. MacDougall, T. C. Chiang, S. L. Cooper, E. Fradkin, and P. Abbamonte, Emergence of charge density wave domain walls above the superconducting dome in $1 T-\mathrm{TiSe}_{2}$, Nat. Phys. 10, 421 (2014).

[46] L. E. Klintberg, S. K. Goh, P. L. Alireza, P. J. Saines, D. A Tompsett, P. W. Logg, J. Yang, B. Chen, K. Yoshimura, and F. M. Grosche, Pressure- and Composition-Induced Structural Quantum Phase Transition in the Cubic Superconductor (Sr, Ca $)_{3} \operatorname{Ir}_{4} \mathrm{Sn}_{13}$, Phys. Rev. Lett. 109, 237008 (2012).

[47] S. S. Saxena, P. Agarwal, K. Ahilan, F. M. Grosche, R. K. W. Haselwimmer, M. J. Steiner, E. Pugh, I. R. Walker, S. R. Julian, P. Monthoux, G. G. Lonzarich, A. D. Huxley, I. Sheikin, D. Braithwaite, and J. Flouquet, Superconductivity on the border of itinerant-electron ferromagnetism in $\mathrm{UGe}_{2}$, Nature (London) 406, 587 (2000).

[48] C.-H. Du, W. J. Lin, Y. Su, B. K. Tanner, P. D. Hatton, D. Casa, B. Keimer, J. P. Hill, C. S. Oglesby, and H. Hohl, X-ray scattering studies of $2 \mathrm{H}-\mathrm{NbSe}_{2}$, a superconductor and charge density wave material, under high external magnetic fields, J. Phys. Condens. Matter 12, 5361 (2000).

[49] Sven Friedemann, Data for publication "Absence of superconducting dome at the charge-density-wave 1 quantum phase transition in 2H-NbSe2" (2020), https://doi.org/10.5523/bris. 3i7a3mzyfbrrp2j871ykh846v0. 